\title{
Study of Point Defect Distributions in Tantalum
}

\author{
A. DjaAfri ${ }^{a}, *$ A. Kadoun ${ }^{b}$, M. Driss-Khodja $^{a}$, A. Elias ${ }^{a}$ And T. DjaAfri ${ }^{c}$ \\ ${ }^{a}$ Laboratoire d'Etudes Physico-Chimiques, Université Tahar Moulay, 20000 Saïda, Algeria \\ ${ }^{b}$ Laboratoire de Microscopie, Microanalyse de la Matière et Spectroscopie Moléculaire, Université Djillali Liabes, \\ 22000 Sidi-Bel-Abbes, Algeria \\ ${ }^{c}$ Département de Physique, Université Tahar Moulay, 20000 Saïda, Algeria
}

(Received January 9, 2017; in final form October 28, 2017)

We have investigated the point defect distributions in tantalum under irradiation by means of the Marlowe code based on the binary collision approximation. The study is carried out by simulating displacement cascades initiated with primary knock-on atom energies ranging from 5 to $20 \mathrm{keV}$. The Molière, Born-Mayer and average modified Lenz-Jensen potentials are used to describe the interactions between tantalum atoms. We have examined the creation of damage, the spatial defects distribution, and the vacancy clustering in tantalum. The results show that with an appropriate recombination radius, less than $16 \%$ of the created defects constitute permanent Frenkel pairs. Spatial configuration of defects indicates a separation between the two point defect types, vacancies and interstitials. The Molière potential favors the production of a greater number of displaced atoms and the development of voluminous cascades more than the other potentials. The cascade volume distributions deviate clearly from a Gaussian distribution. They are large and very stretched toward higher volumes for all used potentials. Only small vacancy clusters are formed in tantalum under irradiation and about $41 \%$ of the produced vacancies are considered as isolated

DOI: 10.12693/APhysPolA.133.39

PACS/topics: 61.82.Bg, 61.80.Ed, 61.72.jj, 61.72.jn

\section{Introduction}

Irradiation of materials by energetic atomic particles leads to the formation of highly disordered regions as a result of displacements of lattice atoms. Such damaged regions, where point defects and clusters of these defects are produced, have significant impact on physical and mechanical properties of materials including hardening, embrittlement, strength, thermal and electrical conductivities. Therefore, understanding the damage production mechanism, the spatial distribution, and clustering of defects are of great practical interest for designing materials to be used in intense irradiation environments [1-3].

Tantalum $\mathrm{Ta}$ is a refractory transition metal. It has a large number of interesting properties including high strength and hardness, good thermal conductivity, good ductility even at low temperatures, and excellent corrosion resistance, that make it a valuable material for a wide range of uses. It is particularly used in high temperature applications such as aircraft engines, in electronic equipments, chemical industry, materials of advanced nuclear energy system, and medical and military fields [4-6].

Considerable efforts have been made over the years to determine the displacement threshold energy value for tantalum $[7,8]$ as well as to develop semi-empirical potential models and enhance our understanding of the mechanical properties of this material [9-13]. However,

*corresponding author; e-mail: djaafri_aek@yahoo.fr there have been very few reports on the damage production in tantalum [14-16]. The displacement cross-section for tantalum irradiated with protons and neutrons at energies up to $1 \mathrm{GeV}$ has been investigated by Broeders and Konobeyev $[14,15]$. In an earlier work [16], we examined the displacement cascades in Ta initiated by primaries of $10 \mathrm{keV}$. We mainly focused on the temporal development of the cascade and effects of temperature and potential on the length distribution of the replacement collision sequences (RCS). We found that RCS were weakly affected by crystal temperature whereas their dependence on the potential was noticeable. Extensive studies have been carried out on damage production and clustering in some bcc metals such as iron, tungsten, and vanadium [17-20]. To the best of our knowledge, there is no work in the literature concerning the spatial configuration of damage and the clustering of point defects in tantalum using the binary collision approximation (BCA) or molecular dynamics models. Therefore, the main purpose of this study is to investigate, via atomistic simulations based on the BCA model, the creation and spatial configuration of point defects, the volume of the damaged region, and the vacancy clustering in irradiated tantalum. These cascade features, not covered in the earlier work [16], would provide further insight into the response of tantalum to irradiation.

In order to study the point defect distributions in tantalum, we have used the BCA model. The computer simulation based on BCA is widely employed to study the interactions of energetic particles with matter. It was successfully applied to backscattering, sputtering, and radiation damage [17, 21-27]. By using BCA calculations, we can cover a wide range of sufficiently high energies, 
treat large numbers of damage events and therefore improve, drastically, cascade statistics [28]. In this model, a cascade is approximated as sequences of binary encounters and the colliding particles are assumed to move along their scattering asymptotes. The displacement cascade starts with the generation of a primary knock-on atom (PKA), which is the target atom displaced by the incoming particle. The PKA continues collisions creating secondary recoil atoms which in turn induce further lattice displacements. In these collision processes, the atoms are dislodged from their location whenever they acquire energy higher than the displacement threshold energy. If the energy of a moving atom is insufficient to create new displacements it comes to rest as interstitial. At the end of the cascade, interstitials and vacancies are produced in equal numbers and the name Frenkel defect is used to describe a stable vacancy-interstitial pair. Not only isolated point defects are generated in a displacement cascade but clusters of vacancies and interstitials can also be constituted. These defects induced by irradiation have a fundamental influence on the physical and mechanical properties of materials.

We will start from computing the number of the displaced lattice atoms in the cascade since a fraction of these atoms will constitute permanent defects. Then, we examine the relative positions of interstitials and vacancies by constructing a distribution function which gives the number of Frenkel pairs as function of the distance vacancy-interstitial. With taking an appropriate recombination radius, we keep only the permanent defects and try to evaluate the volume of the damaged zone by using the principal components analysis. Finally, we will investigate the amount of vacancy clustering and the cluster sizes.

\section{The computational model}

The calculations reported here have been performed with version 15 of the BCA computer code Marlowe. Details concerning the program are described in Refs. [2934]. Only Marlowe aspects related to our work are presented herein. Collision cascades are described as succession of two-body collisions and atoms are supposed to move along straight-line trajectories between collisions. The inelastic atomic collisions constitute of a quasielastic part governed by one of several interatomic potentials available in the Marlowe package and a separate electron excitation part. The classical elastic scattering integrals between the two interacting atoms are evaluated using Gaussian methods. The results are tabulated as functions of the initial kinetic energy of the projectile and the impact parameter in an encounter. They include energies, times, and scattering angles of the colliding atoms. The number of collisions experienced by the projectile before it comes to rest is controlled by a maximum impact parameter value given by the user. Inelastic energy loss is assumed to occur locally by electron excitation [35] and nonlocally according to the Lindhard-Scarff-Schiott
(LSS) theory [36]. Thermal vibrations are included in Marlowe by using a model of uncorrelated Gaussian thermal displacements of the lattice atoms. The distribution variance may be supplied by the user or can be computed from the Debye model [37]. A lattice atom is added to the cascade if it receives a kinetic energy exceeding a displacement energy threshold, $E_{t h}$. To be redisplaced, a nonlattice target must surmount a binding energy $E_{\mathrm{B}}$. If the projectile comes to rest at the recoiling particle site, a replacement event is identified and it is required that the target atom must overcome a binding energy $E_{r}$. When no particle in the cascade remains with energy greater than some cut-off value $E_{c}$, the cascade atoms and lattice sites involved are arranged in the Frenkel pairs.

The crystal tantalum structure is body-centered cubic (bcc) with a lattice parameter $a_{0}=3.304 \AA$ [38]. In our calculations, 5000 primaries were launched isotropically from lattice sites with kinetic energies ranging from 5 to $20 \mathrm{keV}$. The binding energy of the atoms to their lattice sites is taken equal to the cohesive energy in tantalum $E_{t h}=8.09 \mathrm{eV} \mathrm{[39]} \mathrm{and} \mathrm{the} \mathrm{cut-off} \mathrm{energy} \mathrm{is} E_{c}=E_{t h}$. Binding energies for the redisplacement and replacement events were $E_{\mathrm{B}}=0.0 \mathrm{eV}$ and $E_{r}=0.2 \mathrm{eV}$, respectively. Collisions with maximum impact parameter of $0.71 a_{0}$ were evaluated. Thermal displacements of the lattice atoms were described by the Debye model using the Debye temperature of tantalum $T_{D}=240 \mathrm{~K}$ [40]. Calculations were made for temperatures of $300 \mathrm{~K}$ and $1000 \mathrm{~K}$. The interatomic potentials used to describe the interactions between tantalum atoms are the Molière approximation to the Thomas-Fermi potential, the Born-Mayer potential, and the average modified Lenz-Jensen (AMLJ) potential. The Molière potential [41] is given by

$$
V(r)=\left(\frac{Z_{1} Z_{2} e^{2}}{r}\right) \sum_{i=1}^{3} \alpha_{i} \exp \left(-\beta_{i} r / a_{M}\right),
$$

where $Z_{1} e$ and $Z_{2} e$ are the nuclear charges of the projectile and target atoms, respectively, $r$ is the distance between the atoms, $\alpha_{i}=(0.35,0.55,0.10)$ and $\beta_{i}=$ $(0.3,1.2,6.0)$. The screening length $a_{\mathrm{M}}$ is computed in the Marlowe code from the Firsov formula [41]:

$$
a_{\mathrm{M}}=\left(\frac{9 \pi^{2}}{128}\right)^{1 / 3} \frac{a_{\mathrm{B}}}{\left(Z_{1}^{1 / 2}+Z_{2}^{1 / 2}\right)^{2 / 3}},
$$

where $a_{\mathrm{B}}$ is the Bohr radius $(0.529 \AA)$. For Ta-Ta interaction, $Z_{1}=Z_{2}=73$ and $a_{\mathrm{M}}=0.07062 \AA$. The BornMayer potential [41] is governed by

$$
V(r)=A \exp \left(-r / a_{\mathrm{BM}}\right),
$$

where $A=52\left(Z_{1} Z_{2}\right)^{3 / 4}$ and $a_{\mathrm{BM}}$ is the screening length. For the Ta-Ta interaction, $A=32433.08 \mathrm{eV}$ and $a_{\mathrm{BM}}=$ $0.219 \AA$. The average modified Lenz--Jensen potential is written as [42]:

$$
V(r)=\left(\frac{Z_{1} Z_{2} e^{2}}{r}\right) \exp \left(-a_{1} r+a_{2} r \sqrt{r}-a_{3} r^{2}\right),
$$

where $a_{1}=12.3114, a_{2}=9.82485$, and $a_{3}=$ $2.78447[43,44]$. 
It is known that the BCA model predicts an excess of the final number of generated point defects since it does not account for any thermally activated recombination of nearby vacancies and interstitials created in the collisional stage. To remediate the situation, we used in Marlowe a recombination radius $r_{V}[26,45]$. A Frenkel pair is considered to remain stable if the vacancy-interstitial separation distance is larger than $r_{V}$, otherwise, it recombines. We obtained the $r_{V}$ values for different potentials by matching the number of Frenkel pairs computed by Marlowe code to their number calculated from the relation [14]:

$$
N_{F P}(T)=\eta N_{N R T},
$$

where $N_{F P}$ is the number of the Frenkel pairs created by a PKA with energy $T$ in irradiated material, $\eta$ is the defect production efficiency and $N_{N R T}=$ $0.8 T_{d}(T) / 2 E_{d}$ is the number of defects predicted by the Norgett-Robinson-Torrens (NRT) model $E_{d}$ is the effective threshold displacement energy. For tantalum, the average value for $\eta$ is 0.73 and $E_{d}$ is equal to $90 \mathrm{eV}$ [14]. The damage energy denoted $T_{d}$ is computed from the relation [14]:

$$
T_{d}(T)=\frac{T}{1+k\left(3.4008 \varepsilon^{1 / 6}+0.40244 \varepsilon^{3 / 4}+\varepsilon\right)},
$$

where

$$
\begin{aligned}
& k=\frac{32}{3 \pi}\left(\frac{m_{e}}{M_{2}}\right)^{1 / 2} \frac{\left(A_{1}+A_{2}\right)^{3 / 2} Z_{1}^{2 / 3} Z_{2}^{1 / 2}}{A_{1}^{3 / 2}\left(Z_{1}^{2 / 3}+Z_{2}^{2 / 3}\right)^{3 / 4}}, \\
& \varepsilon=\frac{A_{2} T a}{\left(A_{1}+A_{2}\right)\left(Z_{1} Z_{2} e^{2}\right)}, \\
& a=\left(\frac{9 \pi^{2}}{128}\right)^{1 / 3} \frac{a_{\mathrm{B}}}{\sqrt{Z_{1}^{2 / 3}+Z_{2}^{2 / 3}}},
\end{aligned}
$$

$A_{1}$ is the mass number of the PKA, $M_{2}$ and $A_{2}$ are the mass of the target atom and its mass number, $m_{e}$ is the electron mass and $e$ is its charge. The best matching, within the considered range of PKA energies, was found for $r_{V}=4.50 a_{0}, r_{V}=3.75 a_{0}$, and $r_{V}=2.50 a_{0}$ for Molière, Born-Mayer, and AMLJ potentials, respectively.

\section{Results and discussion}

Computing the total number of atoms displaced by a given PKA is an unavoidable task in the study of damage production in irradiated material. Figure 1 shows the mean number of displaced lattice atoms per cascade, $\langle N\rangle$, as function of time for PKA energy of $20 \mathrm{keV}$, for the Molière, Born-Mayer, and AMLJ potentials, at $300 \mathrm{~K}$ and $1000 \mathrm{~K}$ crystal temperatures. Since, at the beginning of the cascade development, the stopped cascade atoms are not yet very numerous, collisions with the lattice atoms are the most frequent events and $\langle N\rangle$ increases with time. This trend persists as long as the mobile atoms are able to eject lattice atoms from their equilibrium positions. As the cascade progresses, the stopped cascade atoms become increasingly potential targets. The kinetic energy of the moving atoms diminishes rapidly being dissipated elastically by producing new recoils or displacing stopped cascade atoms and inelastically in exciting the electrons of the medium. At longer times, the kinetic energy of the recoils is so low that they cannot deliver the requisite binding energy to the lattice atoms to induce new displacements. Accordingly, the number of displaced lattice atoms remains unchanged over time. It is worth to notice that according to previous results [16] relating to the temporal evolution of the number of atoms in motion, this number increases at the same time as $\langle N\rangle$. It attains a maximum when $\langle N\rangle$ begins to reach saturation, then decreases to zero at the end of the cascade since all mobile atoms come to rest. The potential model affects the number of displaced atoms. At the end of the cascade, at the temperature $300 \mathrm{~K}$, $\langle N\rangle$ for the AMLJ potential is about $34 \%$ lower than that for the Molière potential. It appears also that $\langle N\rangle$ is weakly affected by thermal disorder since this number is reduced by only $\approx 2.5 \%$ for all potentials when the crystal temperature is increased from $300 \mathrm{~K}$ to $1000 \mathrm{~K}$.

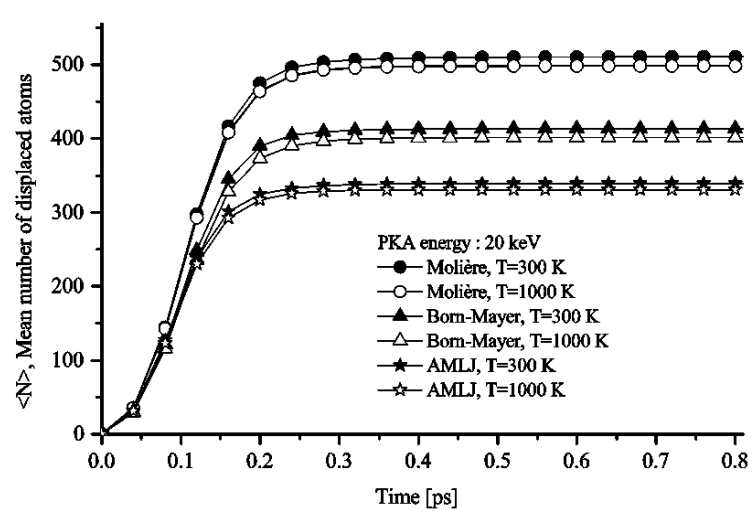

Fig. 1. The mean number of displaced atoms per cascade as function of time. Cascades are generated by $20 \mathrm{keV}$ tantalum atoms at the temperatures $300 \mathrm{~K}$ and $1000 \mathrm{~K}$ for Molière, Born-Mayer, and AMLJ potentials.

The final number of the point defects produced in the cascade depends on the relative positions of vacancies and interstitials created in the ballistic stage. The displaced atoms which come to rest in close proximity to vacancies recombine spontaneously. It is known that the replacement collision sequences (RCS) by carrying atoms far away from vacancies contribute to the damage production. They are favored at low energies and have tendency to be developed along close packed crystallographic directions. Our calculations show that RCS lengths in irradiated tantalum are short. Indeed, the replacement sequences of length three and four constitute about $7 \%$ and $4 \%$, respectively, of the total number of RCS produced with the Molière potential. Thus, only a small fraction of displaced atoms are expelled from the cascade core through RCS and the large proportion of them stay close to vacancies and have the opportunity to re- 


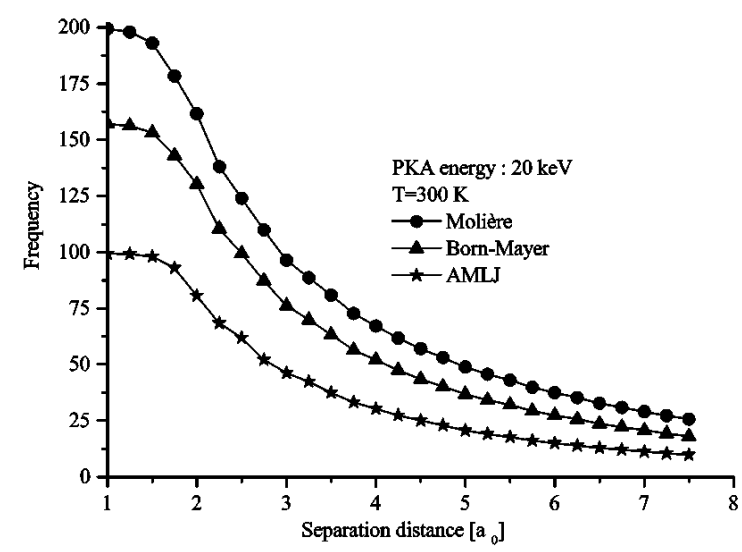

Fig. 2. Mean number of Frenkel pairs created as a function of the vacancy-interstitial recombination distance. Cascades are generated by $20 \mathrm{keV}$ selfbombardment of tantalum at the temperature $300 \mathrm{~K}$ for Molière, Born-Mayer, and AMLJ potentials.

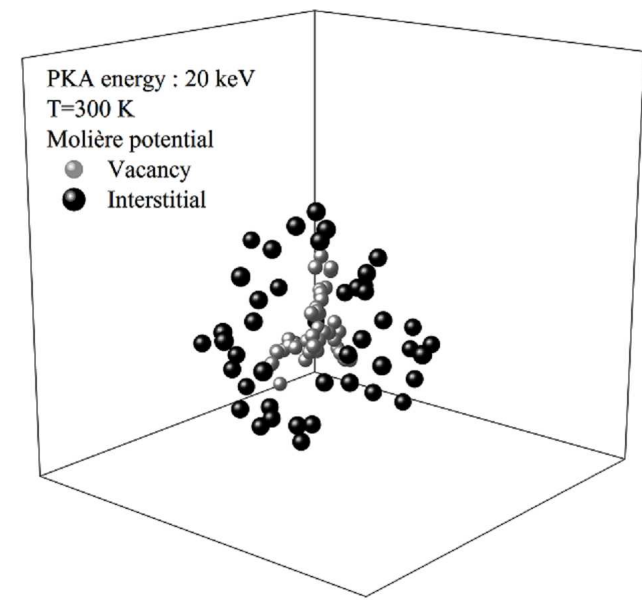

Fig. 3. Spatial configuration of defects produced by a $20 \mathrm{keV}$ tantalum cascade for Molière potential at the crystal temperature of $300 \mathrm{~K}$. Large and dark spheres are interstitials. Small and light spheres are vacancies.

combine. A pertinent study of point defects positions can be done by constructing the distribution function versus the vacancy-interstitial pair-separation distance, $r_{V I}$. It gives the mean number of the Frenkel pairs of separation exceeding $r_{V I}$. Results of such study are presented in Fig. 2 for PKA energy of $20 \mathrm{keV}$ at crystal temperature $300 \mathrm{~K}$. It is clear that small values of the pair-separation distance are sufficient to remove a great number of defects. This confirms that most of the displaced atoms are nearby to vacancies. We obtain by considering the appropriate recombination radius for each potential that the ratio of stable Frenkel pairs over the number of displaced atoms in displacement model with the Molière potential is only $10.4 \%$, while this ratio is about $15.4 \%$ in model with the AMLJ potential.

The question of spatial distribution of vacancies and interstitials in a cascade was treated analytically by Brinkman [46]. He suggested that an energetic particle passing through a material creates not only a collection of point defects, but produces a highly damaged region composed of an outer shell rich in interstitials and a core of vacancies. It is possible, with Marlowe, to record the three spatial coordinates of the permanent point defects positions for illustrating the final spatial configuration of a cascade. Figure 3 exhibits the configuration of defects obtained at the end of $20 \mathrm{keV}$ collision cascade in Ta for the Molière potential at a crystal temperature of $300 \mathrm{~K}$. As it is expected, the spatial configuration indicates a separation between the two point defect types. Vacancies are congregated at the central area of the cascade whereas interstitials are condensed in a halo surrounding this area. The replacement collision sequences play the major role in the eviction of interstitials from the center of the cascade along low-index crystallographic directions and the formation of such configuration.

To evaluate the extent of the damage induced by irradiation of tantalum, we consider that the produced point defects constitute a cloud of points in three-dimensional space and we try to compute the dimensions and the volume of the cascade. To do this, we used the statistical method of principal components analysis [47]. It makes it possible to associate with each displacement cascade an ellipsoid whose directions of principal axes are given by the eigenvectors $\boldsymbol{u}_{1}, \boldsymbol{u}_{2}$, and $\boldsymbol{u}_{3}$ of the centered covariance matrix, $M$, constructed from the point positions in space. The eigenvector $\boldsymbol{u}_{1}$ is associated to the largest eigenvalue of $M$ and $\boldsymbol{u}_{3}$ to the smallest one. The eigenvalues of $M$ are the variances of the point distribution along the principal axes and their square roots, which are the standard deviations, representing the half lengths of these axes. The volume of the ellipsoid which represents the core of the cascade can now be easily computed from the eigenvalues. The use of this procedure for each cascade makes it possible to construct the volume distributions. The core density then can be determined from the ratio of the ellipsoid volume to the number of defect points in the core. Figure 4 shows the cascade volume distributions obtained with $20 \mathrm{keV}$ PKAs, for the Molière and the AMLJ potentials, at a crystal temperature of $300 \mathrm{~K}$. The first thing to be noted is that, regardless the used potential, the asymmetry is a prominent aspect in volume distributions. Statistical quantities, skewness $s$, which characterizes the degree of asymmetry of a distribution relative to its mean, and kurtosis $k$, which describes height and sharpness of the peak with respect to the rest of the data [48], give quantitative information about the shape of the distribution. With the Molière potential, we found $s=7.60$ and $k=108.96$, while with the AMLJ potential, $s=3.20$ and $k=16.84$. Values of the coefficient of variation which is a measure of dispersion are $58 \%$ with respect to the Molière potential and $44 \%$ with respect to AMLJ potential. For the Born-Mayer volume distribution which is not shown in Fig. 4 for readability, we found $s=3.17, k=16.31$, and $38 \%$ for the coefficient of variation. These high values indicate clearly that the distributions deviate from a Gaussian one. Their tails 
are very stretched toward the higher volumes. The cascade volumes are spread out over a wide range of values showing a high dispersion. Hence, we cannot rely on the mean volume to give a meaningful representation of the cascade volume. Even if the cascade is initiated with the same PKA energy and the same interatomic potential, the final cascade configurations can be very different. The loss of correlation between initial conditions and the final cascade configurations was observed in bcc Fe [49]. It was attributed to the thermal displacements [49]. Our results show also that the spatial extent of the damage is potential dependent. Indeed, the Molière potential favors the development of cascades more than the other used potentials.

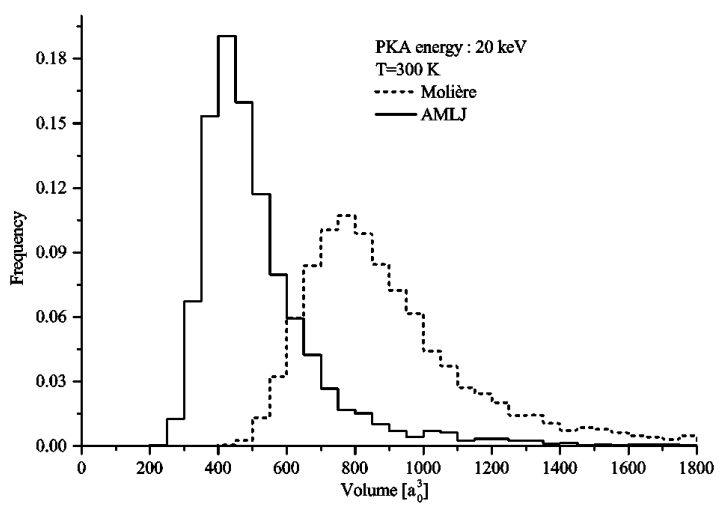

Fig. 4. Cascade volume distributions obtained, with Molière and AMLJ potentials, by a $20 \mathrm{keV}$ tantalum cascade at a crystal temperature of $300 \mathrm{~K}$.

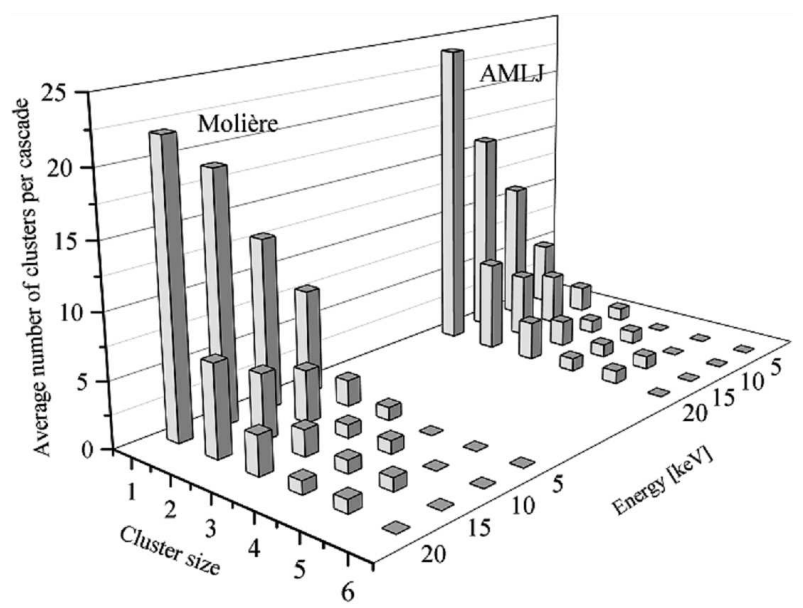

Fig. 5. Average number of vacancy clusters as a function of size and PKA energy for Molière and AMLJ potentials at the crystal temperature $300 \mathrm{~K}$.

Since most of the produced vacancies are concentrated in the cascade core, some of them can form clusters. These irradiation induced defects can influence mechanical and physical properties of the material. Molecular dynamics simulations show that clustering in metals has a noticeable dependence on the crystal structure. Indeed, the defect clustering fraction is higher in metals with fcc structure such as $\mathrm{Cu}, \mathrm{Ni}$ and $\mathrm{Al}$ [50] than in metals with bcc structure. In the present work, we consider that a vacancy is a member of a cluster if it has at least one partner at a distance smaller or equal to the second neighbor distance. Figure 5 depicts the cluster size distribution for vacancies at different PKA energies for the Molière and the AMLJ potentials at temperature of $300 \mathrm{~K}$. The cluster size is defined by the number of vacancies forming the cluster. It comes out from our current statistics that there is no major difference in vacancy clustering when using the two potentials. The amount of clustering and the clusters size rise with rising the PKA kinetic energy. Small vacancy clusters are formed in the cascade. The largest one involves only five point defects in $20 \mathrm{keV}$ PKA energy. The fraction of vacancies considered as isolated is about $41 \%$ for the Molière potential in this energy. This fraction was found about $45 \%$ in previous BCA simulations in bcc Fe [18], and the maximum cluster size was also five vacancies. Molecular dynamics calculations indicate that low vacancy clustering is a behavior present in other irradiated bcc metals such as tungsten [19] and vanadium [20].

\section{Conclusion}

We have used the BCA simulation model to accumulate statistics over 5000 cascades and investigate the defects production, the defects spatial configuration, as well as the vacancy clustering in tantalum. Our results show that most of the displaced atoms come to rest in close proximity to vacancies. Only a small fraction of the displaced atoms constitute permanent Frenkel pairs. The final spatial configuration of permanent defects indicates a separation between vacancies and interstitials. We found, also, that the number of displaced atoms and the cascade extent are influenced by the interatomic potential model. The Molière potential favors the creation of more displaced atoms and the development of voluminous cascades. The cascade volume distributions show a high dispersion. They are large and very stretched toward higher volumes for all used potentials. Small vacancy clusters are formed in tantalum under irradiation and about $41 \%$ of the produced vacancies are considered as isolated.

\section{References}

[1] V.A. Ivchenko, IOP Conf. Ser. Mater. Sci. Eng. 110, 012003 (2016).

[2] C. Lu, K. Jin, L.K. Béland, F. Zhang, T. Yang, L. Qiao, Y. Zhang, H. Bei, H.M. Christen, R.E. Stoller, L. Wang, Sci. Rep. 6, 19994 (2016).

[3] E. Zarkadoula, S.L. Daraszewicz, D.M. Duffy, M.A. Seaton, I.T. Todorov, K. Nordlund, M.T. Dove, K. Trachenko, J. Phys. Condens. Matter 25, 125402 (2013).

[4] R.W. Buckman Jr., JOM 52, 40 (2000). 
[5] Y. Huang, N. Maury, N.X. Zhang, T.G. Langdon, IOP Conf. Ser. Mater. Sci. Eng. 63, 012100 (2014).

[6] J.B. Lambert, Tantalum and tantalum compounds, in: Kirk-Othmer Encyclopedia of Chemical Technology, Wiley-Interscience, 2007, p. 313.

[7] P. Jung, W. Schilling, Phys. Rev. B 5, 2046 (1972).

[8] G. Youngblood, S. Myhra, J.W. DeFord, Phys. Rev. 188, 1101 (1969).

[9] Y. Mishin, A.Y. Lozovoi, Acta Mater. 54, 5013 (2006).

[10] R. Ravelo, T.C. Germann, O. Guerrero, Q. An, B.L. Holian, Phys. Rev. B 88, 134101 (2013).

[11] X.D. Dai, Y. Kong, J.H. Li, B.X. Liu, J. Phys. Condens. Matter 18, 4527 (2006).

[12] Z.L. Liu, L.C. Cai, X.R. Chen, F.Q. Jing, Phys. Rev. B 77, 024103 (2008).

[13] A. Hashibon, A.Y. Lozovoi, Y. Mishin, C. Elsässer, P. Gumbsch, Phys. Rev. B 77, 094131 (2008).

[14] C.H.M. Broeders, A.Yu. Konobeyev, J. Nucl. Mater. 336, 201 (2005).

[15] C.H.M. Broeders, A.Yu. Konobeyev, J. Nucl. Mater. 342, 68 (2005).

[16] A. Djaafri, T. Djaafri, A. Elias, M. Driss Khodja, Afric. Rev. Phys. 8, 365 (2013).

[17] A. Souidi, A. Elias, A. Djaafri, C.S. Becquart, M. Hou, Nucl. Instrum. Methods Phys. Res. B 193, 341 (2002).

[18] A. Souidi, M. Hou, C.S. Becquart, C. Domain, J. Nucl. Mater. 295, 179 (2001).

[19] M.J. Caturla, T.D. de la Rubia, M. Victoria, R.K. Corzine, M.R. James, G.A. Greene, J. Nucl. Mater. 296, 90 (2001).

[20] E. Alonso, M.J. Caturla, T.D. de la Rubia, J.M. Perlado, J. Nucl. Mater. 276, 221 (2000).

[21] W. Takeuchi, Y. Yamamura, Radiat. Eff. 71, 53 (1983).

[22] A. Kuzmichev, V. Perevertaylo, L. Tsybulsky, O. Volpian, J. Phys. Conf. Ser. 729, 012005 (2016).

[23] T.T. Nuver, H. Rudolph, P.A. Zeijlmans van Emmichoven, A. Niehaus, Nucl. Instrum. Methods Phys. Res. B 164-165, 785 (2000).

[24] A. De Backer, A. Sand, C. Ortiz, C. Domain, P. Olsson, E. Berthod, C.S. Becquart, Phys. Scr. T167, 014018 (2016).

[25] A. Elias, M. Driss Khodja, Phys. Proced. 2, 1489 (2009).

[26] M. Hou, C.J. Ortiz, C.S. Becquart, C. Domain, U. Sarkar, A. Debacker, J. Nucl. Mater. 403, 89 (2010).

[27] L. Bukonte, F. Djurabekova, J. Samela, K. Nordlund, S.A. Norris, M.J. Aziz, Nucl. Instrum. Methods Phys. Res. B 297, 23 (2013).
[28] C.P. Race, The Modeling of Radiation Damage in Metals using Ehrenfest Dynamics, Springer Theses, Springer-Verlag, Berlin 2010.

[29] M.T. Robinson, Phys. Rev. B 40, 10717 (1989).

[30] M.T. Robinson, Nucl. Instrum. Methods Phys. Res. B B 48, 408 (1990).

[31] M.T. Robinson, Nucl. Instrum. Methods Phys. Res. B 67, 396 (1992).

[32] M.T. Robinson, Radiat. Eff. 141, 1 (1997).

[33] C.J. Ortiza, A. Souidi, C.S. Becquart, C. Domain, M. Hou, Radiat. Eff. 169, 592 (2014).

[34] M.T. Robinson, MARLOWE: Computer Simulation of Atomic Collisions in Crystalline Solids (Version 15b), RSICC Code Package PSR-137, 2002.

[35] O.S. Oen, M.T. Robinson, Nucl. Instrum. Methods 132, 647 (1976).

[36] J. Lindhard, M. Scharff, H.E. Schiott, K. Danske Vidensk. Selsk., Math.-fys. Medd. 33, 1 (1963).

[37] M. Blackman, Handbuch der Physik, Vol. VII, Part 1, Springer-Verlag, Berlin 1955.

[38] A. Dewaele, P. Loubeyre, M. Mezouar, Phys. Rev. B 70, 094112 (2004).

[39] F.R. De Boer, R. Boom, W.C.M. Mattens, A.R. Miedema, A.K. Niessen, Cohesion in Metals: Transition Metal Alloys, North-Holland, Amsterdam 1989.

[40] C. Kittel, Introduction to Solid State Physics, 8th ed., Wiley, 2004.

[41] W. Eckstein, Computer Simulation of Ion-Solid Interaction, in: Springer Series in Materials Science, Springer-Verlag, Berlin 1991.

[42] M.T. Robinson, in: Fundamental Processes in Sputtering of Atoms and Molecules, SPUT92, Ed. P. Sigmund, Roy. Dan. Acad., Copenhagen 1992, p. 37.

[43] S.T. Nakagawa, Y. Yamamura, Radiat. Eff. 105, 239 (1988).

[44] S.T. Nakagawa, Radiat. Eff. 116, 21 (1991).

[45] G. Vizkelently, S.M. Foiles, Nucl. Instrum. Methods Phys. Res. B 371, 111 (2015).

[46] J.A. Brinkman, Am. J. Phys. 24, 246 (1956).

[47] M. Hou, Phys. Rev. A 39, 2817 (1989).

[48] G. Bohm, G. Zech, Introduction to Statistics and Data Analysis for Physicists, Ed. Deutsches Elektronen-Synchrotron, 2010.

[49] M. Hou, A. Souidi, C.S. Becquart, J. Phys. Condens. Matter 13, 5365 (2001).

[50] G.S. Was, in: Fundamentals of Radiation Materials Science: Metal and Alloys, Springer-Verlag, Berlin 2007, p. z298. 